\title{
Atrial fibrillation and risk of stroke in dialysis patients
}

\author{
James B. Wetmore, MD, MS ${ }^{1,2}$, Edward F. Ellerbeck, MD, MPH $^{3}$, Jonathan D. Mahnken, \\ $\mathrm{PhD}^{4}$, Milind Phadnis, $\mathrm{PhD}^{4}$, Sally K. Rigler, MD, MPH${ }^{5,6}$, Purna Mukhopadhyay, $\mathrm{PhD}^{4}$, \\ John A. Spertus, MD, MPH${ }^{7}$, Xinhua Zhou, $\mathrm{PhD}^{4}$, Qingjiang Hou, MS ${ }^{4}$, and Theresa I. \\ Shireman, $\mathbf{P h D}, \mathbf{R P h}^{2,3}$ \\ ${ }^{1}$ Department of Medicine, Division of Nephrology and Hypertension, University of Kansas School \\ of Medicine, Kansas City, KS, USA \\ ${ }^{2}$ The Kidney Institute, University of Kansas School of Medicine, Kansas City, KS, USA \\ ${ }^{3}$ Department of Preventive Medicine and Public Health, University of Kansas School of Medicine, \\ Kansas City, KS, USA \\ ${ }^{4}$ Department of Biostatistics, University of Kansas School of Medicine, Kansas City, KS, USA \\ ${ }^{5}$ Department of Medicine, University of Kansas School of Medicine, Kansas City, KS, USA \\ ${ }^{6}$ The Landon Center on Aging, University of Kansas School of Medicine, Kansas City, KS, USA \\ ${ }^{7}$ St. Luke's Mid America Heart Institute, University of Missouri-Kansas City, Kansas City, MO, \\ USA
}

\section{Abstract}

Purpose-Both stroke and chronic atrial fibrillation (AF) are common in dialysis patients, but uncertainty exists in the incidence of new strokes and the risk conferred by chronic AF.

Methods-A cohort of dually-eligible (Medicare \& Medicaid) incident dialysis patients was constructed. Medicare claims were used to determine the onset of chronic AF, which was specifically treated as a time-dependent covariate. Cox proportional hazards models were used to model time to stroke.

Results-Of 56,734 patients studied, 5629 (9.9\%) developed chronic AF. There were 22.8 ischemic and 5.0 hemorrhagic strokes per 1000 patient-years, a ratio of approximately 4.5:1. Chronic AF was independently associated with time to ischemic (HR 1.26, 99\% CI's 1.06-1.49, $P=0.0005)$, but not hemorrhagic, stroke. Race was strongly associated with hemorrhagic stroke: African-Americans (HR 1.46, 99\% CI's 1.08 - 1.96), Hispanics (HR 1.64, 99\% CI's 1.16 - 2.31), and others (HR 1.76, 99\% CI's $1.16-2.78)$ had higher rates than did Caucasians $(P<0.001$ for all).

\section{(C) 2012 Elsevier Inc. All rights reserved.}

Corresponding author: James B. Wetmore, MD, MS, Division of Nephrology \& Hypertension, 3901 Rainbow Blvd., MS 3002, Kansas City, KS 66160; Phone 913-588-6076; FAX 913-588-3867; jwetmore@kumc.edu.

Disclosures: The authors have no conflicts of interest to declare.

Disclaimer: The data reported here have been supplied by the United States Renal Data System (DUA\#2007-10 \& 2009-19) and the Centers for Medicare \& Medicaid Services (DUA\#19707). The interpretation and reporting of these data are the responsibility of the author(s) and in no way should be seen as an official policy or interpretation of the U.S. government.

Publisher's Disclaimer: This is a PDF file of an unedited manuscript that has been accepted for publication. As a service to our customers we are providing this early version of the manuscript. The manuscript will undergo copyediting, typesetting, and review of the resulting proof before it is published in its final citable form. Please note that during the production process errors may be discovered which could affect the content, and all legal disclaimers that apply to the journal pertain. 
Conclusions-Chronic AF has a significant, but modest, association with ischemic stroke. Race/ethnicity is strongly associated with hemorrhagic strokes. The proportion of strokes due to hemorrhage is much higher than in the general population.

\section{Keywords}

stroke; atrial fibrillation; dialysis; end stage renal disease; Medicare; Medicaid

\section{Introduction}

Stroke is a catastrophic health event and a leading cause of disability. In the general population, atrial fibrillation (AF) is a well-established risk factor for stroke [1]. We have recently reported that $7 \%$ of prevalent dialysis patients have chronic AF [2] (a rate ten-fold greater than that of the general population [3] ), a finding which might suggest that chronic AF could be a major contributor to the stroke burden suffered by dialysis patients. Conversely, dialysis patients have a high burden of vascular disease, diabetes and hypertension, all of which could conceivably modify the contribution of AF to stroke. As such, the contribution of AF to stroke in chronic dialysis patients is uncertain. Understanding the relationship between chronic AF and stroke in this unique population is particularly important given recent evidence which suggests that the risks of warfarin for stroke prophylaxis in AF may outweigh its benefits in dialysis patients [4, 5].

An additional issue of uncertainty is the rate of various stroke types in chronic dialysis patients. Reported stroke rates range from 15 [6-9] up to nearly $50[4,10]$ events per 1000 patient-years. Similarly, the reported rates of stroke subtypes differ substantially. While in the general population the ratio of ischemic to hemorrhagic strokes is about 6-7:1[11,12], in dialysis patients this ratio reportedly varies from one comparable to that of the general population $(\sim 6: 1[5,10,11])$ to one in which hemorrhagic strokes are distinctly common ( $3: 1)[4,9]$. These findings suggest that improved characterization of stroke rates and types is needed for the dialysis population, in particular a determination of the differential rates of strokes during periods of chronic AF versus during "AF-free" time.

In the present study, we used a large cohort of incident, dually-eligible (Medicare-Medicaid) chronic dialysis patients to investigate two important stroke-related questions. First, utilizing new information on the sensitivity and specificity of stroke-related ICD-9 (International Classification of Disease) claims in identifying strokes from Medicare data [13], we determined ischemic and hemorrhagic stroke rates in chronic dialysis patients and divided these into at-risk (chronic AF-time) and non-at-risk (AF-free) times. Second, we determined the association of chronic $\mathrm{AF}$ and a broad spectrum of other covariates on ischemic, hemorrhagic, and all (combined) strokes using a recently-reported chronic AF identification strategy [2] as well as a rigorous, time-varying approach to model AF. Finally, to compare results of our dually-eligible population to that of other populations $[4,14]$, we tested whether the $\mathrm{CHADS}_{2}$ risk stratification scheme used in the general population to predict stroke risk in patients with $\mathrm{AF}$ [15] would perform well in broadly risk-stratifying duallyeligible chronic dialysis patients. A more complete understanding of all these issues is critically important for evaluating the risks and benefits of potential therapies, such as longterm anticoagulation, for dialysis patients who have chronic AF.

\section{Materials and Methods}

\section{Study design and data sources for analysis}

We performed a retrospective cohort analysis of incident, dually-eligible (Medicare-

Medicaid) chronic dialysis patients. We examined dually-eligible dialysis patients for two 
reasons. First, we have a long-standing interest in this population, which represents a particularly vulnerable group of medically-needed individuals who are more likely to be indigent, female, and minority than general dialysis population[16]. Second, the definition of chronic AF relied, in part, on drug prescribing information which is readily available only in Medicaid-eligible individuals (see below for more details). The data sources and strategy used for linking Medicare and Medicaid patients have been previously described [2]; details are in Supplementary Appendix 1.

\section{Study cohort and rationale for analytic approach}

The cohort consisted of individuals over 18 years of age who initiated chronic dialysis on or after January 1, 2000, who survived at least 90 days after dialysis initiation, and who were continuously enrolled in Medicare and Medicaid from dialysis initiation until October 2, 2005 (permitting a minimum of 90 days of follow-up before December 31, 2005). Briefly, to ensure that all Medicare claims were observable, we studied only individuals who were both dually-eligible from the time of dialysis initiation and who had Medicare was the primary payer; individuals were censored if they lost either Medicare or Medicaid eligibility (i.e., at the first coverage gap). Patients enrolled in any form of managed care plan (e.g., Medicaid managed care plans such as in Arizona or Tennessee) or in the Department of Veterans Affairs health system were excluded since claims data were not available. Of note, persons on chronic dialysis were generally not enrolled in Medicare managed care plans prior to 2006. Additional triggers for censoring were receipt of a kidney transplant or death on or before 12/31/05.

\section{Covariates and descriptive variables}

A variety of covariates were considered, a priori, as potential risk factors for stroke. Demographic and clinical variables, drawn from the CMS 2728 dialysis intake form; details on the covariates and their definitions are described in Supplementary Appendix 1. Because previous stroke is a strong predictor of future stroke, we examined the cohort for stroke claims in the first 90-day run-in period prior to the start of the observation window; an individual was considered to have preexisting stroke if either a history of a cerebrovascular accident (CVA) was declared on the CMS 2728 form or if a stroke claim appeared during the 90-day run-in period. Strokes occurring after this period (i.e., during the observation window) were considered "incident" strokes.

Additionally, we supplemented the medical information from the CMS 2728 form with a modified form of the Liu Comorbidity Index (a summary measure of comorbidity burden) [17]; more details are provided in Supplementary Appendix 1.

\section{Determination of chronic AF}

We specifically sought to study the association of persistent or recurrent (and therefore chronic) $\mathrm{AF}$, rather than transient $\mathrm{AF}, \mathrm{AF}$ due to structural heart disease, or AF due to reversible or treatable medical or surgical causes $[3,18,19]$. The strategy used to determine chronic AF utilized the ICD-9 code 427.31 and has been previously described in detail [2]; further details are in Supplementary Appendix 1. Because we sought to determine risk of stroke in AF-time and AF-free time, we treated chronic AF as a time-varying condition: when the definition of chronic $\mathrm{AF}$ was met, $\mathrm{AF}$ was then dated from the time of the first qualifying claim.

\section{Stroke outcomes}

Outcomes were assessed from Medicare claims. We modeled time to first stroke in the observation period. We utilized new information on the sensitivity and specificity of stroke- 
related ICD-9 claims to identifying strokes from Medicare data [13]. A specific approach, in which only the codes with the highest specificity for ischemic or hemorrhagic strokes were used, was the primary approach. A sensitive approach, which used a broader range of stroke codes, was selected as a sensitivity analysis. For the specific approach for ischemic strokes, the strategy of Go et al was utilized [19]. The appearance of codes 433, 434, or 436 in the primary position of an inpatient claim was first assessed; if these were not present, the presence of code 362.3 was sufficient to attribute an ischemic stroke. (This code, for retinal vascular occlusion, is sometimes used when cerebrovascular events produce visual symptoms[3].) If the 433,434 , or 436 were present and accompanied by a fatal hospitalization, an ischemic stroke was attributed. If 434, 434, or 436 were present and the hospitalization was nonfatal but the hospitalization duration was $\geq 48$ hours, an ischemic stroke was attributed, while if the hospitalization was $<48$ hours, the presence of a carotid endarterectomy (CEA, ICD-9 procedure code 38.12) was assessed. If a CEA was not present an ischemic stroke was attributed, while the presence of a CEA in a hospitalization $<48$ hours meant that a stroke code was not counted. (A code for an ischemic stroke, even when non-acute, is sometimes used to justify a carotid endarterectomy, so employment of this code as described above can reduce misclassification of stroke residua as acute strokes.) The sensitive approach differed only by treating code 435 analogously to 433,434 , and 436 .

For the specific approach to hemorrhagic strokes, the presence of codes 430 or 431 in an inpatient claim residing in the first (primary) position was sufficient to attribute a hemorrhagic stroke; the sensitive approach added codes that some investigators use [13] for hemorrhagic strokes, namely 432, .852.0, 852.2, 852.4, or 853 . These codes will capture more intracranial bleeds, but likely also capture bleeds due to trauma.

\section{Statistical analysis}

After generation of descriptive statistics, we used Cox proportional hazards modeling was to determine the risk factors associated with new stroke; separate models were created for ischemic stroke, hemorrhagic stroke, and all (combined) strokes in the case of both the primary models and the sensitivity analyses described above. AF was treated as a timedependent covariate such that every stroke was designated as occurring either in the absence of chronic AF ("AF-free time") or during chronic AF ("AF-time"), if AF developed. To determine the raw rates of stroke per unit of person-time, the number of strokes were summed and divided by the at-risk time. Finally, we sought to determine whether the $\mathrm{CHADS}_{2}$ risk scores[15] permitted effective risk stratification for ischemic stroke in duallyeligible dialysis patients. Scores were calculated for each patient, who were then grouped into one of three strata (scores of 0 or 1,2, and $\geq 3$ ). Modeling occurred only after patients developed chronic AF. As this was an exploratory analysis, we determined bivariate hazard ratios (HRs) for ischemic stroke for each of the three strata and determined whether HRs differed significantly.

All models satisfied Cox proportionality assumptions, and we examined for goodness-of-fit. We utilized a $P$-value of $<0.01$ to be the threshold for statistical significance. This approach allowed us to reduce the likelihood of a type I error with (presumably) minimal impact on the power to detect significant finding given the large sample size. All statistical analyses were done with SAS 9.2 (Cary, NC, USA).

\section{Compliance and protection of human research participants}

The research protocol was approved by the institutional review board at the University of Kansas Medical Center (KUMC). The work was undertaken in accordance with the principles of the Declarations of Helsinki. Data Use Agreements (DUA) between KUMC and the USRDS and CMS were in place. 


\section{Results}

Figure 1 shows the construction of the study cohort. There were a total of 62,812 duallyeligible individuals who initiated dialysis between January 1, 2000 and October 2, 2005 (i.e., who survived at least 90 days prior to our final date of Dec 31, 2005). We then eliminated individuals who had valvular heart disease or hyperthyroidism as well as the small number who had incomplete information, leaving 56,734 individuals in the final study sample.

The characteristics of the cohort are shown in Table 1, and bivariate differences between individuals who had ischemic stroke (our primary outcome of interest) and those who did not are shown. Individuals who had ischemic strokes were significantly older (by about 4.5 years), more likely to be female, more likely to be African-American or Hispanic, less likely to be smokers or substance abusers, more likely to have physical function impairments, and more likely to have diabetes and cardiovascular comorbidities (hypertension, coronary artery disease, history of strokes, and peripheral vascular disease) as well as to develop atrial fibrillation. As would be expected, they were observed for a significantly period of time (15.6 versus 22.1 months).

\section{Ischemic stroke}

Using specific criteria for ischemic stroke, there were 2,348 total ischemic strokes in 103,080 patient-years (py), or 22.8 per 1000 py. Strokes were then stratified into having occurred in chronic AF time or in AF-free time; stroke rates were 22.2 per 1000 py in AFfree time and 28.2 per 1000 py during chronic AF time. The attributable risk of being in chronic $\mathrm{AF}$ was therefore 6.0 strokes per 1000 py, and the attributable risk percent was $6.0 \div$ 26.2, or $21.3 \%$. These stroke rates are shown in Table 2.

Factors associated with new ischemic strokes are shown in Table 3. Chronic AF, treated as a time-dependent covariate, was significantly associated with ischemic stroke (hazard ratio [HR] 1.26, 99\% confidence intervals [CI's] $1.06-1.49, P=0.0005)$. Additionally, increasing age (HR per decade 1.24, 99\% CI's 1.20 - 1.31), female sex $(1.39,1.23$ - 1.56), diabetes $(1.40,1.23-1.59)$, and history of a previous stroke $(1.33,1.14-1.55)$ were all significantly $(P<0.0001)$ associated with ischemic stroke. Individuals with the highest BMI $\left(>30 \mathrm{~kg} / \mathrm{m}^{2}\right)$ had significantly fewer strokes $(0.81,0.71-0.94, P=0.0002)$ compared to individuals in the referent BMI category $\left(20-24.99 \mathrm{~kg} / \mathrm{m}^{2}\right)$. For African-Americans and Hispanics, race/ethnicity was not associated with ischemic stroke (HR $1.02[0.90-1.16]$ and 0.86 [0.73 - 1.01], respectively), although there was a decreased likelihood for individuals of "other" races $(0.76(0.59-0.98), P=0.005)$.

To test the robustness of our findings, several sensitivity analyses were performed. An identical analysis, save for elimination of the modified Liu Comorbidity Index in the modeling, found time-dependent chronic AF to be associated with a slightly higher HR for ischemic stroke of 1.37 (99\% CI's, $1.16-1.62, P<0.0001$ ); the risks associated with diabetes and a previous stroke increased very slightly as well (not shown). A second sensitivity analysis was performed in which ischemic stroke was classified using a more sensitive definition; the total number of strokes increased by $11.9 \%$, to 2,627 . The overall annual ischemic stroke rate increased to 25.6 per 1000 py (Table 2), driven by slight increases in stroke rates in both the AF-free time (24.9 per 1000 py) and chronic AF time (31.3 per 1000 py). In this scenario, the attributable risk was similar to the previous one (6.4 per 1000 py), as was the attributable risk percent (20.4\%). Results of this model (not shown) were virtually identical to the original model, with increasing age, female sex, diabetes, and history of a previous stroke all remaining highly significant $(P<0.0001)$ and the HRs changing, at most, by 0.1 . Time-dependent chronic AF showed virtually the same 
association with ischemic stroke in this more sensitive model $(1.22,1.04-1.43, P=$ $0.0017)$.

\section{Hemorrhagic strokes}

Using specific criteria for hemorrhagic stroke, there were 529 total hemorrhagic strokes, or 5.0 per 1000 py (Table 2). There were 493 hemorrhagic strokes in chronic AF-free time (5.3 per 1000 py) and only 36 hemorrhagic strokes in chronic AF-time (3.3 per 1000 py). The time-standardized rate of total ischemic to hemorrhagic strokes was about 4.5:1, using comparable specific approaches. Thus, hemorrhagic strokes comprised approximately $18 \%$ of the total strokes.

Modeling hemorrhagic stroke (Table 3) revealed no effect of age, sex, diabetes, previous stroke, or time-dependent chronic AF. As was the case for ischemic strokes, the highest category of BMI was inversely associated with the outcome $(0.54,0.40-0.74, P<0.0001)$. While the aim of the present study was not examine the association of race with stroke per se, nevertheless the most salient finding of this model was the effect of race: compared to Caucasians, each minority group had a substantially increased risk of hemorrhagic strokes, ranging from an HR of 1.46 for African-Americans to 1.76 for members of other races, which suggests that the risk for various stroke subtypes is intimately connect to race and ethnicity in some way. This finding has also been noted in the general population, in which African-Americans were at a 50\% increased risk of stroke compared to Caucasian patients[20]; however, comparisons between the dialysis and non-dialysis populations should be considered cautiously, as the populations differ in many critically-important ways.

A sensitivity analysis was performed in which hemorrhagic stroke was assessed using a more sensitive stroke definition. This resulted in a $92 \%$ increase in time-standardized events. As shown in Table 2, in absolute terms the rate of hemorrhagic strokes increased to 9.7 per 1000 py, but there was still not a large difference in rates based on AF status (9.9 per 1000 py in AF-free time and 7.6 per 1000 py in chronic AF-time). In this more sensitive model, age was now weakly associated with hemorrhagic stroke $(1.09$ per decade, $1.03-1.16, P=$ 0.0003). Similar to the specific hemorrhagic stroke model, time-dependent chronic AF, sex, diabetes, and history of a previous stroke remained unassociated with the outcome, while again BMI $>30 \mathrm{~kg} / \mathrm{m}^{2}(P<0.0001)$ and substance abuse $(P=0.0009)$ remained associated. Overall, the effect of non-Caucasian race was attenuated, with African-American $(P=0.11)$ and Hispanic race/ethnicity $(P=0.013)$ showing a trend for more hemorrhagic strokes, and with "other" race still demonstrating a frankly significant effect $(P<0.0001)$.

\section{Combined strokes}

Overall (ischemic or hemorrhagic) stroke rate was 27.3 per 1000 py (Table 2); this was not divided into chronic $\mathrm{AF}$ and $\mathrm{AF}-$ free time since previous analysis showed that chronic $\mathrm{AF}$ increases the risk only for ischemic strokes. A single model, using specific definitions of both ischemic and hemorrhagic stroke, was constructed (Table 3). Overall, this model seemed driven primarily by the effects of factors on ischemic strokes. Time-dependent AF was again significantly associated with overall stroke $(1.21,1.03-1.42, P=0.0019)$. Age (HR 1.02 per year), female sex (1.32), diabetes (1.35), and previous stroke (1.28) were all significantly $(P<0.0001)$ associated with time to any stroke, while high BMI was significantly inversely associated with the outcome $(0.76, P<0.0001)$.

All of the above models satisfied the assumptions of Cox Hazards proportionality. 


\section{Ischemic stroke risk stratification}

Three strata of $\mathrm{CHADS}_{2}$ were modeled $(0-1,2$, and $\geq 3)$ using the specific definition of ischemic stroke. This is shown as Figure 2, which is the time to first ischemic stroke in surviving dialysis patients with chronic atrial fibrillation stratified by $\mathrm{CHADS}_{2}$ score. There were significant differences between each of the 3 curves, in which individuals with lower scores had longer times to stroke (test for trend, $P<0.0001$ ). Compared to the lowest stratum, the HR for stroke of the middle stratum was $1.89(P<0.0001)$ and for the highest stratum was $2.82(P<0.0001)$; compared to the middle stratum, the HR for the highest stratum was $1.49(P<0.0001)$

\section{Discussion}

In this study, we used a large cohort of incident dually-eligible chronic dialysis patients to determine the rate of new ischemic and hemorrhagic strokes and the risk of stroke conferred by chronic AF. To assure rigor in our analysis, we treated chronic AF as a time-dependent covariate and also classified strokes using both sensitive and specific approaches. We found that there were 22.8 ischemic, 5.0 hemorrhagic, and 27.3 total strokes per $1000 \mathrm{py}$. We also found that, after adjustment for a broad range of factors, the increased risk of chronic AF to ischemic and total strokes was 1.26 and 1.21 , respectively, in dialysis patients.

The present report represents the largest investigation of the association between chronic $\mathrm{AF}$ and stroke in chronic dialysis patients to date, helping to establish firm estimates for the magnitude of the association between chronic AF and stroke. Much previous work has been limited by issues of sample size, ascertainment and determination of $\mathrm{AF}$, and analytical treatment of chronic AF. For example, Toyoda et al [7] provided a comprehensive picture of strokes in an incident cohort followed prospectively over a maximum of 22 years. However, while they had rich clinical data on the types and anatomical locations of strokes, there were only 151 stroke events to analyze. They found that AF conferred a risk of approximately 2.8 for carotid territorial infarcts, but it appears as though AF was not treated as a timedependent covariate (i.e., that AF was assessed only at baseline). Sanchez-Perales et al [21] assessed AF both at baseline and during followup in an analysis of incident patients in a single program and found an attributable risk for stroke of 3.1, but the number of patients was relatively modest $(n=449)$ and it is unclear how the development of AF was modeled. Knoll et al [8] reported that the risk of stroke in AF was approximately 2.1-fold higher than in the absence of AF, but again, number of patients was small $(n=235)$, as was the total number of events; only 4 strokes occurred in patients with AF. An additional report from Power et al [9] examined a sizable cohort of patients $(n=2384)$ and used medical records to establish the presence of a stroke and classify its subtype, but they used a prevalent population; interestingly, $\mathrm{AF}$ was not associated with stroke.

The most comprehensive published report to date originates from the Dialysis Outcomes and Practice Patterns Study, in which >17,500 patients were assessed for AF both at baseline and during the observation period [14]. These investigators found that AF conferred a risk of 1.79 for subsequent stroke, but it is possible that transient or non-sustained AF might have caused misclassification since a single diagnosis of AF upon hospitalization was apparently used to classify individuals as having chronic AF henceforth during follow-up; this may have caused over-attribution of the association between chronic AF and outcomes, a shortcoming overcome in our design. Indeed, this may be the reason that our estimate of the contribution of AF to ischemic stroke is somewhat smaller than other studies. The very large size of our study sample, combined with our ability to precisely date strokes as well as to rigorously operationalize the timing of onset of chronic AF, may enable us to determine a more precise quantification of the risk of stroke associated with chronic AF in the dialysis population 
It is important to note that virtually all studies report AF to be a relatively less important contributor to stroke in the dialysis population than is the case in the general population. Foundational work from the Framingham cohort over 20 years found AF to increase the risk of stroke by about four-fold [1], an association several-fold higher than that reported across the literature in dialysis. While we cannot entirely dismiss the possibility that differences in study designs across populations is the reason for such findings, it seems far more likely that the phenomenon of "competing risks" is operative. That is, because the general population is substantially healthier than the dialysis population, AF may be a relatively more important risk factor for stroke in the former. Even after covariate adjustment, the general ill-health of the dialysis population, much of which is due to cardiovascular disease, may mean that many strokes are the result of processes independent of AF, making chronic AF a relatively less important stroke risk factor.

One noteworthy finding was an inverse association, after adjustment for other factors, between hypertension and stroke in our study. This may be unexpected given the direct correlation between hypertension and stroke in the general population. However, studies of prevalent dialysis patients demonstrate that many are characterized by a distinct physiology, namely the controversially-termed phenomenon of 'reverse epidemiology' [22]. This may explain how hypertension, listed as a CMS 2728 comorbidity, could be associated with a decreased likelihood of stroke. Many individuals develop end stage renal disease on the backdrop of a long history of cardiovascular disease which, by the time they reach dialysis, may have reached advanced stages. Many individuals with advanced cardiovascular disease demonstrate left ventricular hypertrophy and heart failure, and, as such, may not have sufficient cardiac reserve to manifest hypertension. These same patients might also have the highest burden of cerebrovascular disease and, as such, be particularly prone to stroke. Whether this phenomenon is responsible for our findings deserves further exploration.

Our model for hemorrhagic strokes differed substantially from that for ischemic strokes. The most salient finding of this model was the effect of race: compared to Caucasians, each minority group had a substantially increased risk of hemorrhagic strokes, ranging from an HR of 1.46 for African-Americans to 1.76 for members of other races, which suggests that the risk for various stroke subtypes is intimately connect to race and ethnicity in some way. The association of race with differential risk for hemorrhagic stroke does not appear to have been systematically explored in the prior literature, although reports from Japan consistently show a much higher ratio of hemorrhagic to ischemic strokes compared to reports from Western countries $[6,7,23,24]$. Since a history of a CVA was not significantly associated with a hemorrhagic stroke, our findings might suggest that, unlike ischemic strokes, hemorrhagic strokes could be sudden, catastrophic events, perhaps brought on by some interplay between race and other, as-yet-uncertain, factors. If replicated, these findings warrant further investigation.

Both the overall rates of strokes as well as the ratio of ischemic to hemorrhagic strokes we found are in the middle of the range reported in the literature; as such, our very large study sample helps to firmly establish these estimates. While study designs differ, reports in Asian dialysis patients typically report ischemic-to-hemorrhagic ratios of only about $1.5-2.5$ to 1 $[6,7,23,24]$, while in non-Asian patients, the ratios reported range from $3: 1$ [9] to approximately $6: 1[10,11]$. As such, our ratio of roughly $4.5: 1$ is in the middle of the ranges reported outside of Asia. As might be expected, event rates also vary in the literature, from a low of about 12.3 - 14.9 total stroke events per 1000 py[7-9] up to $42-49$ per 1000 py [10, 25]. Our combined event rate of about 27 events per 1000 py is broadly comparable to those in reports of Sanchez-Perales et al [21] and Wizemann et al [14] (24 and 34 events per 1000 py, respectively). 
There are limitations in our work which are inherent to any observational study. A form of selection bias is undoubtedly present since only dually-eligible patients, who differ in important ways from non-dually-eligible patients [16], were studied; this reduces the generalizability of our findings. Thus our results should be extrapolated only with extreme caution. Information bias may well be present as well. For example, we cannot rule out the possibility that individuals with atrial fibrillation may be more likely to have stroke recorded as an ICD-9 diagnosis code, which might artificially inflate the association between atrial fibrillation and stroke. The possibility also exists that residual confounding may be present. Although we tried to identify all confounders present in the administrative dataset, there may still be other, unmeasured confounders that at play; for example, in our findings relating race to hemorrhagic stroke, race could simply be a marker of another unmeasured confounder such as degree of control of hypertension.

We also did not have information on anticoagulation status, so future work stratifying patients into those exposed to warfarin and those not is important. As with other large-scale studies, we relied on claims data to infer stroke events, and we lacked information from medical records. However, to bolster confidence in our findings, we used contemporary approaches to claims-based stroke identification and performed multiple sensitivity analyses; these alternate approaches did not materially alter our findings.

In conclusion, we used a time-varying approach to determine the risk of chronic AF for stroke in large cohort of incident dialysis patients. Chronic AF is associated with a modest risk for ischemic, but not hemorrhagic, strokes. The $\mathrm{CHADS}_{2}$ scoring system appears to have reasonable ability to risk-stratify dually-eligible individuals with chronic AF for ischemic stroke. Stroke rates during periods of chronic AF as well as in AF-free time have been ascertained. Non-Caucasians have significantly greater risks for hemorrhagic strokes than did Caucasians, a finding that deserves further exploration.

\section{Supplementary Material}

Refer to Web version on PubMed Central for supplementary material.

\section{Acknowledgments}

The authors thank Connie Wang, MD, and Amanda Gellhaus for technical assistance with manuscript preparation.

Sources of Funding: Funding for this study was provided by NIH (NIDDK) grants K23 DK085378-01 (J.B.W.) and R01 DK080111-02 (T.I.S.), by a National Kidney Foundation Young Investigator Award (J.B.W.), and by a Sandra A. Daugherty Foundation Grant (J.B.W.).

\section{References}

1. Wolf PA, Abbott RD, Kannel WB. Atrial fibrillation as an independent risk factor for stroke: the Framingham Study. Stroke; a journal of cerebral circulation. 1991; 22(8):983-8. Epub 1991/08/01.

2. Wetmore JB, Mahnken JD, Rigler SK, Ellerbeck EF, Mukhopadhyay P, Spertus JA, et al. The prevalence of and factors associated with chronic atrial fibrillation in Medicare/Medicaid-eligible dialysis patients. Kidney Int. 2012; 81(5):469-76. Epub 2011/12/23. [PubMed: 22189842]

3. Go AS, Hylek EM, Phillips KA, Chang Y, Henault LE, Selby JV, et al. Prevalence of diagnosed atrial fibrillation in adults: national implications for rhythm management and stroke prevention: the AnTicoagulation and Risk Factors in Atrial Fibrillation (ATRIA) Study. Jama. 2001; 285(18):23705. [PubMed: 11343485]

4. Chan KE, Lazarus JM, Thadhani R, Hakim RM. Warfarin use associates with increased risk for stroke in hemodialysis patients with atrial fibrillation. J Am Soc Nephrol. 2009; 20(10):2223-33. [PubMed: 19713308] 
5. Winkelmayer WC, Liu J, Setoguchi S, Choudhry NK. Effectiveness and safety of warfarin initiation in older hemodialysis patients with incident atrial fibrillation. Clin J Am Soc Nephrol. 2011; 6(11): 2662-8. Epub 2011/10/01. [PubMed: 21959598]

6. Iseki K, Fukiyama K. Predictors of stroke in patients receiving chronic hemodialysis. Kidney Int. 1996; 50(5):1672-5. Epub 1996/11/01. [PubMed: 8914035]

7. Toyoda K, Fujii K, Fujimi S, Kumai Y, Tsuchimochi H, Ibayashi S, et al. Stroke in patients on maintenance hemodialysis: a 22-year single-center study. Am J Kidney Dis. 2005; 45(6):1058-66. Epub 2005/06/16. [PubMed: 15957135]

8. Knoll F, Sturm G, Lamina C, Zitt E, Lins F, Freistatter O, et al. Coumarins and survival in incident dialysis patients. Nephrol Dial Transplant. 2012; 27(1):332-7. Epub 2011/07/21. [PubMed: 21771750]

9. Power A, Chan K, Singh SK, Taube D, Duncan N. Appraising stroke risk in maintenance hemodialysis patients: a large single-center cohort study. Am J Kidney Dis. 2012; 59(2):249-57. Epub 2011/09/29. [PubMed: 21944665]

10. Sozio SM, Armstrong PA, Coresh J, Jaar BG, Fink NE, Plantinga LC, et al. Cerebrovascular disease incidence, characteristics, and outcomes in patients initiating dialysis: the choices for healthy outcomes in caring for ESRD (CHOICE) study. Am J Kidney Dis. 2009; 54(3):468-77. Epub 2009/04/21. [PubMed: 19376618]

11. Seliger SL, Gillen DL, Longstreth WT Jr, Kestenbaum B, Stehman-Breen CO. Elevated risk of stroke among patients with end-stage renal disease. Kidney Int. 2003; 64(2):603-9. [PubMed: 12846756]

12. Rosamond W, Flegal K, Furie K, Go A, Greenlund K, Haase N, et al. Heart disease and stroke statistics--2008 update: a report from the American Heart Association Statistics Committee and Stroke Statistics Subcommittee. Circulation. 2008; 117(4):e25-146. Epub 2007/12/19. [PubMed: 18086926]

13. Andrade SE, Harrold LR, Tjia J, Cutrona SL, Saczynski JS, Dodd KS, et al. A systematic review of validated methods for identifying cerebrovascular accident or transient ischemic attack using administrative data. Pharmacoepidemiol Drug Saf. 2012; 21(Suppl 1):100-28. Epub 2012/01/25. [PubMed: 22262598]

14. Wizemann V, Tong L, Satayathum S, Disney A, Akiba T, Fissell RB, et al. Atrial fibrillation in hemodialysis patients: clinical features and associations with anticoagulant therapy. Kidney Int. 2010; 77(12):1098-106. Epub 2010/01/08. [PubMed: 20054291]

15. Gage BF, Waterman AD, Shannon W, Boechler M, Rich MW, Radford MJ. Validation of clinical classification schemes for predicting stroke: results from the National Registry of Atrial Fibrillation. Jama. 2001; 285(22):2864-70. Epub 2001/06/13. [PubMed: 11401607]

16. Wetmore JB, Rigler SK, Mahnken JD, Mukhopadhyay P, Shireman TI. Considering health insurance: how do dialysis initiates with Medicaid coverage differ from persons without Medicaid coverage? Nephrol Dial Transplant. 2010; 25(1):198-205. [PubMed: 19736241]

17. Liu J, Huang Z, Gilbertson DT, Foley RN, Collins AJ. An improved comorbidity index for outcome analyses among dialysis patients. Kidney Int. 2010; 77(2):141-51. [PubMed: 19907414]

18. Go AS, Hylek EM, Borowsky LH, Phillips KA, Selby JV, Singer DE. Warfarin use among ambulatory patients with nonvalvular atrial fibrillation: the anticoagulation and risk factors in atrial fibrillation (ATRIA) study. Annals of internal medicine. 1999; 131(12):927-34. [PubMed: 10610643]

19. Go AS, Hylek EM, Chang Y, Phillips KA, Henault LE, Capra AM, et al. Anticoagulation therapy for stroke prevention in atrial fibrillation: how well do randomized trials translate into clinical practice? Jama. 2003; 290(20):2685-92. [PubMed: 14645310]

20. Howard VJ, Kleindorfer DO, Judd SE, McClure LA, Safford MM, Rhodes JD, et al. Disparities in stroke incidence contributing to disparities in stroke mortality. Annals of neurology. 2011; 69(4): 619-27. Epub 2011/03/19. [PubMed: 21416498]

21. Sanchez-Perales C, Vazquez E, Garcia-Cortes MJ, Borrego J, Polaina M, Gutierrez CP, et al. Ischaemic stroke in incident dialysis patients. Nephrol Dial Transplant. 2010; 25(10):3343-8. Epub 2010/05/15. [PubMed: 20466665] 
22. Kalantar-Zadeh K, Block G, Horwich T, Fonarow GC. Reverse epidemiology of conventional cardiovascular risk factors in patients with chronic heart failure. Journal of the American College of Cardiology. 2004; 43(8):1439-44. [PubMed: 15093881]

23. Iseki K, Fukiyama K. Clinical demographics and long-term prognosis after stroke in patients on chronic haemodialysis. The Okinawa Dialysis Study (OKIDS) Group. Nephrol Dial Transplant. 2000; 15(11):1808-13. Epub 2000/11/10. [PubMed: 11071969]

24. Kawamura M, Fijimoto S, Hisanaga S, Yamamoto Y, Eto T. Incidence, outcome, and risk factors of cerebrovascular events in patients undergoing maintenance hemodialysis. Am J Kidney Dis. 1998; 31(6):991-6. Epub 1998/06/19. [PubMed: 9631844]

25. Kuo CC, Lee CT, Ho SC, Kuo HW, Wu TN, Yang CY. Haemodialysis and the risk of stroke: A population-based cohort study in Taiwan, a country of high incidence of end-stage renal disease. Nephrology (Carlton). 2012; 17(3):243-8. Epub 2011/12/17. [PubMed: 22171843]

26. Wetmore JB, Mahnken JD, Mukhopadhyay P, Hou Q, Ellerbeck EF, Rigler SK, et al. Geographic variation in cardioprotective antihypertensive medication usage in dialysis patients. Am J Kidney Dis. 2011; 58(1):73-83. [PubMed: 21621889] 


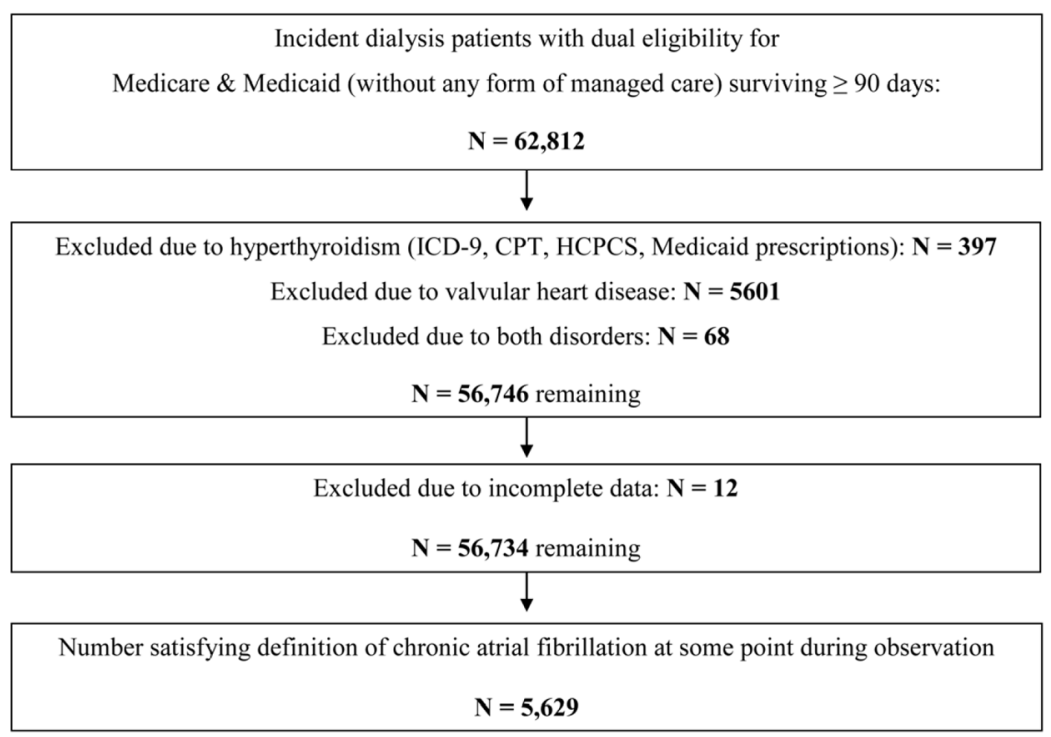

ICD-9, International Classification of Diseases $-9^{\text {th }}$ Revision; CPT, Common Procedural

Terminology; HCPCS, Healthcare Common Procedural Coding System.

Figure 1.

Construction of the study cohort 


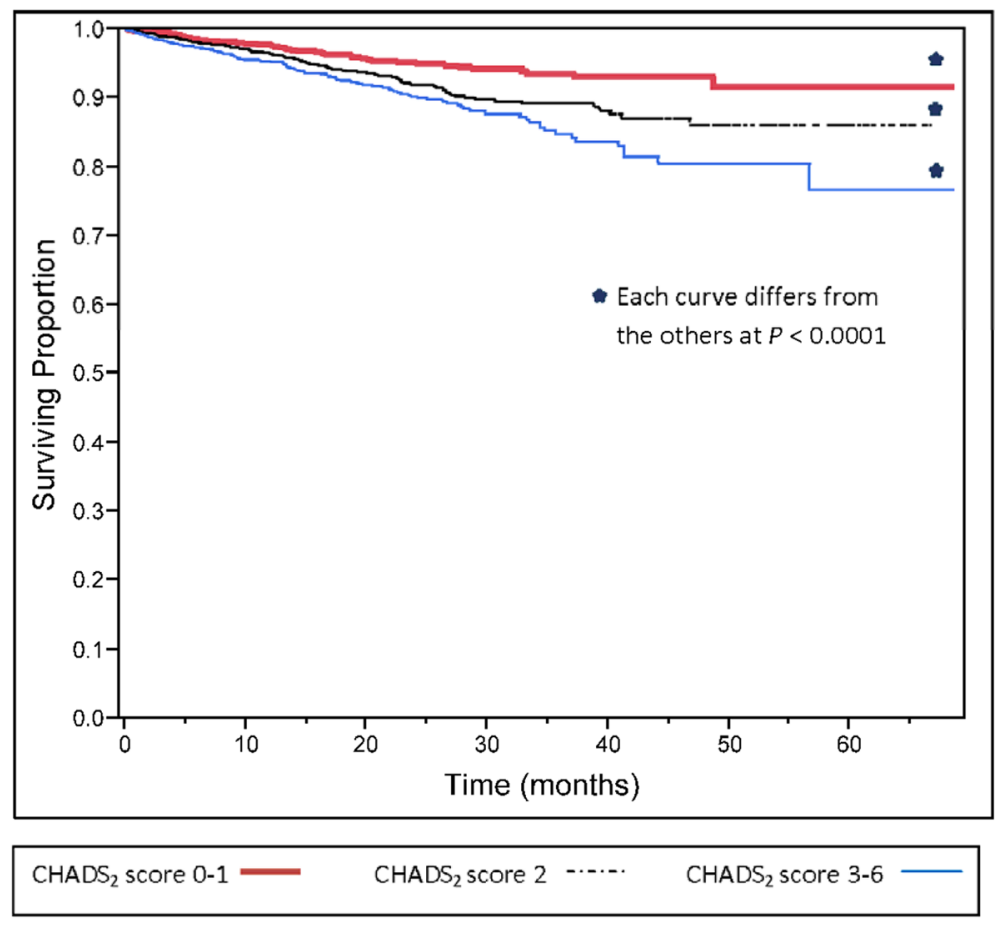

Figure 2.

Time to first ischemic stroke in surviving dialysis patients with chronic atrial fibrillation, by stratum of $\mathrm{CHADS}_{2}$ score. 


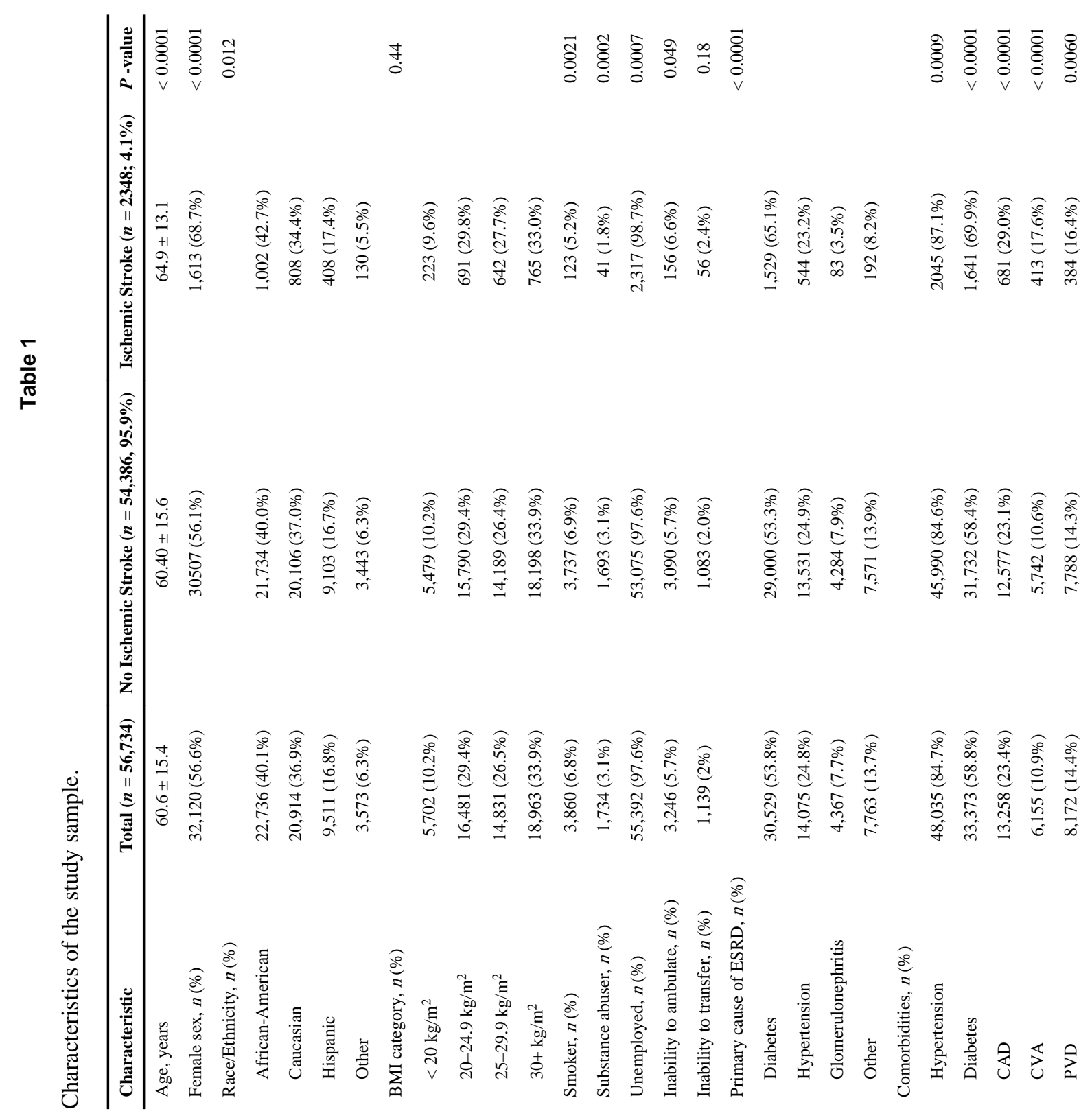

Ann Epidemiol. Author manuscript; available in PMC 2014 March 01. 


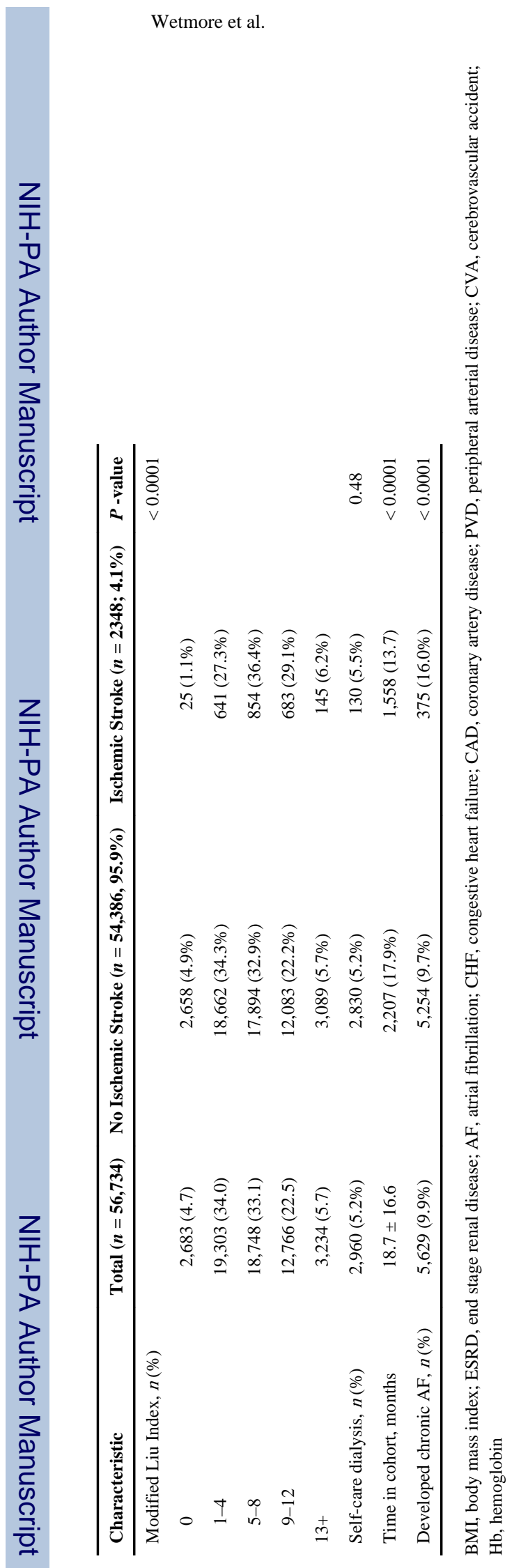

Ann Epidemiol. Author manuscript; available in PMC 2014 March 01. 
Table 2

Stroke rates stratified by stroke type and identification strategy.

\begin{tabular}{|c|c|c|c|}
\hline \multirow[b]{2}{*}{ Stroke Scenario } & \multicolumn{3}{|c|}{ Strokes per 1000 patient-years } \\
\hline & Total & AF time & AF-free time \\
\hline \multicolumn{4}{|l|}{ Ischemic } \\
\hline Specific definition & 22.8 & 28.2 & 22.2 \\
\hline Sensitive definition & 25.6 & 31.3 & 24.9 \\
\hline \multicolumn{4}{|l|}{ Hemorrhagic } \\
\hline Specific definition & 5.0 & 3.3 & 5.3 \\
\hline Sensitive definition & 9.7 & 7.6 & 9.9 \\
\hline \multicolumn{4}{|l|}{ Ischemic + Hemorrhagic } \\
\hline Specific definition & 27.3 & - & - \\
\hline
\end{tabular}

$\mathrm{AF}$, chronic atrial fibrillation 
